ISSN electrónico: 2602-8069

\title{
LA COMUNICACIÓN COMO ESTRATEGIA DE MERCADOTECNIA SOCIAL PARA MINIMIZAR EL IMPACTO DE LA CONTAMINACIÓN EN EL COMEDOR UNIVERSITARIO, DE LA UNIVERSIDAD ESTATAL DE BOLÍVAR
}

Communication as a social marketing strategy to minimize the impact of pollution on the university dining room, of the Bolívar State University

\section{Elsita Margoth Chávez García}

Carrera de Mercadotecnia. Facultad de Ciencias Administrativas,

Gestión Empresarial e Informática. Universidad Estatal de Bolívar, Guaranda, Ecuador. elmarchaga@hotmail.com

Carmen Patricia Chávez García

Ingeniera en Administración de Empresas de la Universidad Tecnológica Equinoccial (UTE- Quito). Maestrante de Dirección de Negocios en Aden University.

pchavez@hotmail.com

Giovanny Lennin Haro Sosa

Carrera de Gastronomía y Promoción y Cuidados de la Salud.

Escuela Superior Politécnica de Chimborazo. Riobamba, Ecuador.

giovalenin@hotmail.com

Fecha de recepción del artículo: 09/09/2019

Fecha de aceptación definitiva: 06/11/2019 


\section{RESUMEN}

La presente investigación analiza un problema de orden mundial, que involucra no solo a las autoridades e instituciones de una ciudad, sino a todas las personas que realizan prácticas comunes de consumo y que se relacionan con el uso indiscriminado de plásticos. Se planteó como objetivo identificar las formas de contaminación por el uso de plásticos a través de encuestas a los consumidores y expendedores en la Universidad de Bolívar para así, diseñar una alternativa de consumo que los sustituya y puedan ser implementadas en el comedor universitario, otro objetivo fue concientizar a los usuarios y proveedores del servicio de alimentación sobre la importancia de preservar el medio ambiente a través de estrategias de consumo sostenibles y amigables. Para ello se realizó una investigación cualitativa y cuantitativa, en donde se describieron las características del fenómeno a través de los datos obtenidos en las encuestas y en la observación. Esto permitió diseñar la propuesta alternativa de solución al problema detectado sobre la influencia del plástico en la contaminación ambiental, el que se espera disminuir reemplazando los envases y contendores de plástico por cartón y otros materiales biodegradables. Este proyecto se espera ejecutarlo en dos etapas, la primera que dará paso a la introducción de una nueva propuesta de servicio con recursos amigables con el medio ambiente, cómodos y de bajo costo. La segunda etapa consiste en la socialización de los principales resultados obtenidos.

Palabras clave: Mercadotecnia social, comunicación, contaminación, concientización, alternativa.

\section{ABSTRACT}

The present investigation is related to a worldwide problem, which involves not only the authorities of a city, canton, or institutions, but all the people who carry out common consumption practices and that are related to the indiscriminate use of plastics. The objective was to identify the forms of contamination by the use of plastics through surveys of users and consumers, followed by designing a consumption alternative that replaces them and can be implemented in the university canteen, in addition to raising awareness among users and suppliers. Food service on the importance of preserving the environment through sustainable and friendly consumption strategies. For this, the type of qualitative and quantitative research was used, where the characteristics of the phenomenon were described through the data obtained in the surveys and observation. This allowed us to design the alternative solution proposal to the problem detected on the influence of plastic on environmental pollution, which is expected to be reduced by replacing plastic containers and containers with cardboard and other biodegradable ones. What is expected to be carried out through two stages, the first one that will give way to the introduction of a new service proposal with environmentally friendly, comfortable and low-cost resources. The second stage consists in the socialization of the main results obtained.

Keywords: Social marketing, communication, pollution, awareness, alternative. 


\section{INTRODUCCIÓN}

En el mundo la contaminación ambiental es generada, entre otros factores, por el consumo indiscriminado de plásticos y por su liberación, esto es objetos no biodegradables en espacios naturales, que suponen un riesgo para la salud de los seres vivos que habitan los ecosistemas contaminados, incluyendo a plantas, animales y seres humanos (Linea Verde Ceuta, 2018). El plástico se ha considerado como factor contaminante del medio acuático y terrestre, ya que al llegar a dichos medios no se desintegra fácilmente. Para Aguilar (2016) la contaminación ambiental es la incorporación a los cuerpos receptores de sustancias sólidas, líquidas o gaseosas, o mezclas de ellas, siempre que alteren desfavorablemente las condiciones naturales del mismo, o que puedan afectar la salud, la higiene o el bienestar del público.

La producción global de plásticos se ha disparado en los últimos 50 años, y en especial, en las últimas décadas, entre 2002 y2013 aumentó un 50 \%: de 204 millones de toneladas en 2002, a 299 millones de toneladas en 2013, donde China es el principal productor de plásticos seguido de Europa, Norteamérica y Asia (excluyendo ya a China), según Estévez (2019), quien también menciona que dentro de Europa, más de dos tercios de la demanda de plásticos se concentran en cinco países: Alemania (24.9\%), Italia (14.3\%), Francia (9.6 $\%)$, Reino Unido (7.7 \%) y España $(7.4 \%)$.

En América Latina, la tasa media de generación de basura per cápita es de $0.87 \mathrm{~kg}$ al día, lo que supera el promedio mundial fijado en $0.74 \mathrm{~kg}$. En todo el mundo, los países de ingreso alto generan más del $34 \%$ de los desechos, pese a que en ellos solo vive el 16 $\%$ de la población del planeta, se estima que mientras un pueblo es pobre, menos produce basura, según la BBC (2018). Para Salinas (2011) los desechos plásticos son uno de estos factores que están constituidos por macromoléculas producidos por transformaciones de sustancias naturales o síntesis directa a partir de productos extraídos del petróleo, del gas natural, del carbón o de otras materias minerales, denominado polimeración, que provocan daños al medio.

Hasta el año 2012 en Ecuador se producían 61117 toneladas de basura semanales, de ellas solo el $20 \%$ se llevaba a espacios adecuados para su tratamiento, el resto iba a vertederos a cielo abierto, botaderos controlados, botaderos en vías, quebradas y ríos. El $53.9 \%$ era material orgánico, el $24.4 \%$ cartón y papel, el $10.9 \%$ plástico, el $6.5 \%$ metales y chatarra, y el $4.4 \%$ vidrio. La producción de residuos per cápita en Ecuador era de $0.81 \mathrm{~kg}$ por habitante y por día. Los cantones con mayor población total son también los cantones con mayor producción diaria de residuos sólidos. En el Ecuador, únicamente el $52 \%$ de los municipios realiza la recolección diferenciada de los residuos hospitalarios. De estos solamente el $24 \%$ lo dispone en una celda especial; el porcentaje restante lo hace directamente en el relleno sanitario o en el botadero público (Morán, 2018).

Para Morán de acuerdo con el estudio realizado por la investigadora en temas de salud, María Fernanda Solís en el año 2016, un $4.1 \%$ de los cantones bota su basura a los ríos. Cuando esto ocurre, los desechos pueden viajar cientos de kilómetros y llegar hasta el mar, ocasionando un problema más grave, puesto que los desechos como el plástico se desintegren y lleguan a la cadena alimenticia, lo cual afecta la salud de las especies. Según sus cifras, el $95 \%$ de la contaminación marina es microplástico, un tóxico que es ingerido por el plancton, peces y otras criaturas marinas que luego son consumidas por el ser humano.

En la actualidad, por las actividades diarias la gente se ha acostumbrado a llevar cosas en envases desechables, incluso no es raro que la ropa sea desechable, por tanto, la comida es desechable, esto genera y promueve una cultura de desecho constante. El estilo 
de vida moderno nos hace pensar a las personas que hay que ser prácticos y rápidos en las cosas que hacemos, que no queda tiempo para nada y que el hecho de lavar, reutilizar y otras actividades tendientes a la economía y conservación de los recursos es cada vez menos importante.

En los lugares de consumo masivo se han adoptado estrategias de servicio al cliente que minimizan el tiempo de espera, ahorran el presupuesto que se paga a los empleados para que no laven platos y utensilios, y se podría decir que esta práctica les otorga un falso prestigio al entregar la comida en recipientes o envases plásticos. Uno de estos lugares es el comedor universitario ubicado en la Universidad Estatal de Bolívar, en donde utilizan envases, fundas, vasos y utensilios plásticos para vender la mayoría de sus alimentos, por ser fáciles de conseguir, su costo es bajo y efectiviza el servicio, volviendo más rápida la atención al cliente.

La universidad por su parte no cuenta con estrategias de gestión a nivel comercial en los centros de consumo y expendio de alimentos masivos, tales como bares, comedores y otros similares, esto deviene en que la gran cantidad de basura que se genera a diario no cuente con ningún sistema de contención, clasificación o de reciclaje, provocándose un daño importante al medio ambiente por la falta de conciencia, información e interés de la comunidad estudiantil sobre el tema. La utilización de plásticos no ha parado y la contaminación aumentá, convirtiéndose el consumo de plásticos en el factor número uno, ya que al llegar a ríos y mares se descompone en microplásticos que pasan a formar parte de la cadena alimenticia de peces y animales marinos que en algún momento pueden llegar a ser consumidos por el ser humano.

En la tesis titulada: "Análisis de los desechos de botellas plásticas de los habitantes de bastión popular de Guayaquil", se concluye que las personas conocen el daño que realizan al desechar plásticos al ambiente, pero no practican la reutilización de este material, por lo que es necesario promover el reciclaje en las escuelas y colegios para fomentar una cultura ecológica, analizando los beneficios del reutilizamiento de los desperdicios y formalizarlo, explorando nuevos mercados de utilización (Chicaiza y González, 2016).

La mayor parte de los ciudadanos conocen el impacto que provoca desechar plásticos, pero no hacen nada al respecto y continúan botando en cualquier contenedor mesclando desechos orgánicos, plásticos, vidrios, cartón y papel, generando contaminación que, según los autores Pérez y Merino (2008) es un material que al introducirse en un medio cualquiera constituye un elemento contaminante. El uso más habitual del término se produce en el ámbito de la ecología con lo que se conoce como contaminación ambiental, que es la presencia en el ambiente de cualquier agente (físico, químico o biológico) en lugares, formas y concentraciones que pueden ser nocivos para la salud, la seguridad o para el bienestar de la población.

Una vez descrito el problema de investigación, se hace necesario introducir al Marketing y sus diferentes estrategias, como un medio de sensibilización para quienes hacen uso del comedor estudiantil. En primera instancia, acorde a la definición proporcionada por American Marketing Association (2016) "El marketing es la actividad, un conjunto de instituciones y procesos para crear, comunicar, entregar, e intercambiar ofertas que tienen valor para los consumidores, clientes, socios y la sociedad en general".

De forma que, se busca sensibilizar el comportamiento voluntario de un grupo de la población se adopta al Marketing social, como una de las partes específicas de actuación, que en palabras de Góngora (2014) en su artículo denominado: "Mercadotecnia social: una herramienta necesaria para la promoción de salud”, señala la definición de Kottler (2006) en 
donde menciona que, "la mercadotecnia social es una estrategia para el cambio de la conducta y combina los mejores elementos de los enfoques tradicionales al cambio social en un marco integrado de planeación y acción, al tiempo que utiliza avances en la tecnología de las comunicaciones y en las técnicas de comercialización".

En el análisis y tomando como referencia al anterior concepto citado, es meritorio que las estrategias de Marketing Social empleadas sean participadas en medios efectivos de comunicación, mediante un plan estratégico, como aplicación de un modo específico en las relaciones humanas, que puede consistir en relaciones de participación recíproca o de comprensión, de interacción humana con los otros o el conjunto de formas que puede adoptar la convivencia (Vera y Villegas, 1997).

Se ha destinado el comedor universitario como lugar de investigación, mismo que está ubicado en la Universidad Estatal de Bolívar, del cantón Guaranda, provincia de Bolívar, durante el período comprendido entre abril de y agosto de 2019. Los beneficiarios directos son los usuarios de los productos y servicios que se ofrecen en el comedor, quienes podrán contar con envases y utensilios amigables con el medio ambiente, libres de contaminación, sanitarios, y de rápida descomposición, que no cause daños con la naturaleza.

Lo que se desea mitigar es el impacto de la contaminación ambiental por el uso de plásticos en el comedor universitario de la Universidad de Bolívar en el período de abril a agosto de 2019, mediante el "Diseño y promoción de un proyecto de reciclaje de plástico y protección del medio ambiente", sabiendo que, los estudiantes no reciclan y, por ende, no reutilizan. La utilización de folletos, entre otras estrategias, incentiva un poco a crear conciencia para disminuir los desechos y reutilizar. El comedor universitario de la Universidad Estatal de Bolívar, está ubicado en el sector sur oeste de la institución, junto a la Biblioteca General, cuenta con seis bares al interior, mismos que dan una atención a los usuarios desde las 9:00 hasta las 18:00 de la tarde.

El público o comensales que utilizan el servicio de alimentación son generalmente estudiantes de las diferentes facultades, personal administrativo y público en general que no se agrupan por categorías, sino que asisten al lugar a servirse alimentos, en horarios diferentes y con gustos diversos. Entre los menús que ofrecen están: sánduches, ensalada de chochos, almuerzos, encebollados, alimentos envasados, gaseosas, jugos, aguas, entre otros. Los recipientes en los que se sirven son envases de plástico, botellas con agua, refresco y otras bebidas, fundas y tarrinas de plástico transparente, cucharas, platos y vasos desechables, bandejas y otros materiales que están hechos básicamente con este material o con otros derivados de petróleo. Las prácticas de recolección de basura son generales, no se realiza el reciclaje, clasificación o reutilización de materiales que pueden disminuir los niveles de contaminación.

Para evitar o minimizar el impacto contaminante causado directamente por los hábitos del hombre se vuelve necesario crear una campaña de Marketing Social, con la intención de cambiar el comportamiento voluntario de los miembros de la sociedad, mediante campañas de información, reciclaje, reutilización y demostración. Para (Pérez y Gardey, 2013) el reciclaje implica dar una nueva vida al material en cuestión, lo que ayuda a reducir el consumo de recursos y la degradación del planeta. La base del reciclaje se encuentra en la obtención de una materia prima o producto a partir de un desecho. Un bien ya utilizado (como una botella de plástico vacía) puede destinarse a la basura o reciclarse y adquirir un nuevo ciclo de vida (al derretir el plástico y utilizarlo en la fabricación de una nueva botella, por ejemplo). Según Eva (2009) la reutilización de productos es cuando le damos una nueva utilidad a un producto que se ha desechado porque ya no sirve para su uso original (esto quiere decir que 
ya ha concluido su vida útil original), contribuyendo a mejorar el medio ambiente, puesto que así se reduce la producción de nuevos bienes que demanden recursos naturales y energía, y también se evita la generación de residuos. En el ámbito de los residuos urbanos, se habla de las tres "R" que caracterizan una gestión ambientalmente correcta de estos desechos, a saber: reducción, reutilización y reciclaje.

\section{METODOLOGÍA}

Se utilizó una investigación mixta, con un enfoque y diseño cualitativo, cuantitativo y descriptivo. Lo cualitativo según Díaz (2018) es parte del estudio de fenómenos o personas que emiten opiniones sobre un escenario social y cultural del cual forman parte. Considerando que la investigación se realizó a un grupo de personas que forman parte del fenómeno medioambiental.

El enfoque de la investigación fue cualitativa y cuantitativa, ya que se identificó las prácticas más comunes de la contaminación a partir del uso de plásticos de los usuarios y proveedores de servicios alimenticios en el comedor universitario y se categorizó, para poder visualizar mediante una apreciación en términos de frecuencias y porcentajes.

El diseño cualitativo permitió establecer relaciones entre los datos recogidos y los observados a partir de cálculos matemáticos obtenidos como consecuencia de un fenómeno QuestionPro (2019). Como el objeto de investigación es la contaminación que se genera por el consumo de plásticos en el comedor universitario, el diseño cualitativo permitió realizar una valoración de dichos niveles (cantidad de basura, tipo de envases utilizados, entre otros) con lo que se podrá tener una visión de su impacto en el medio ambiente.

A través del diseño cuantitativo los investigadores pudieron llegar a inferir conclusiones a partir de datos procesables que permitieron visualizar soluciones y generar la toma de decisiones basadas en números y su análisis (QuestionPro, 2019). Mediante la ayuda de encuestas y la aplicación de estándares que permitieron medir los niveles de contaminación a partir del uso de plásticos se presentó cuadros estadísticos y análisis porcentuales que generen una visión general del problema para plantear soluciones aplicables por usuariosproveedores del servicio y amigables con la naturaleza.

También se utilizó un diseño descriptivo como puntal para la presentación de datos, delimitando las cusas y consecuencias del problema en cuestión (Rodríguez, 2018). Con el diseño descriptivo, se realizó una caracterización del fenómeno investigado, a través de identificar las causas y consecuencias del uso indiscriminado de plásticos, para proponer soluciones al mismo.

El tipo de investigación fue aplicada, la cual hace énfasis a la investigación de problemas que se les puede atribuir soluciones a través de teorías generales en un momento dado, después de haber determinado las características y su interés en la solución del mismo según (Vargas, 2009). Una vez que se conocen las características del fenómeno investigado, se estima su aplicación en el diseño, propuesta y aplicación de estrategias de consumo que sustituya a los plásticos y puedan ser adoptadas por los usuarios y proveedores del servicio de alimentación en el comedor universitario.

Se aplicaron encuestas a los usuarios y prestadores de servicios en el comedor universitario de la Universidad Estatal de Bolívar, en el momento de la compra de sus alimentos, con el fin de conocer: el nivel de consumo de productos que llevan plástico en los envases, porta alimentos y menaje; el nivel de conocimiento sobre el tema de contaminación y la necesidad de fortalecerlos para disminuir su impacto en el medio ambiente, esto a através 
de la aplicación de un cuestionario, elaborado a base de preguntas cerradas y opción múltiple, diseñadas con base en las variables identificadas: la contaminación ambiental en el comedor universitario; como variables independiente y dependiente, respectivamente.

A través del método de observación sencilla se procedió a identificar la cantidad de usuarios del comedor universitario, el tipo de plásticos que se consumen en el lugar, las formas de eliminación de los desechos, y otros que están ligados con el tema. Esta técnica se implementó en dos momentos de la investigación, el primero para determinar por simple inspección la cantidad de usuarios por día en el comedor, lo que permitió extraer la muestra. El segundo momento fue para determinar el tipo de plásticos que se consumen en el lugar (envases, porta alimentos, menaje, etc.), las formas de eliminación de los desechos y otros que sean definidos como necesarios. Se utilizó una guía de observación, misma que permitió registrar los datos observados.

Para obtener la muestra se aplicó la siguiente fórmula (1); propuesta por Paredes (2009) para poblaciones finitas, en donde la población es conocida.

$$
\begin{aligned}
& \mathrm{n}=\boldsymbol{N} \\
& \mathrm{E} 2(\mathrm{~N}-1)+1
\end{aligned}
$$

En dónde, después de aplicar los datos, se obtuvo $\mathrm{n}=367.11$, es decir 367 personas a encuestar.

\section{RESULTADOS}

Se realizó una observación no participativa durante tres horas en diferentes momentos del día, lo que estipuló las siguientes cantidades de personas que asistieron a los locales en el comedor.

Tabla 1. Expendedores del comedor universitario

\begin{tabular}{|l|l|l|}
\hline \multicolumn{1}{|c|}{ Nombre del ofertante de comida } & \multicolumn{1}{c|}{ Horas pico } & \multicolumn{1}{c|}{ Horas bajas } \\
\hline Local "Doña Vale" & 76 comensales & 7 \\
\hline Local "Charito's Bar" & 44 comensales & 8 \\
\hline Local "Bar Silvi's" & 22 comensales & 4 \\
\hline Local "Chester's" & 24 comensales & 5 \\
\hline Local “El Rey Camarón Marisquería" & 7 comensales & 1 \\
\hline Subtotal & 173 & 25 \\
\hline Total, de comensales (Horas pico + Horas bajas) & & 198 \\
\hline Promedio (Total de comensales/2) & 99 \\
\hline
\end{tabular}

Elaboración: Los Investigadores.

A los 99 comensales promedio por día, se multiplican por 9 horas de servicio y cinco días hábiles a la semana que atienden en el comedor, dando un total de 4455 comensales por semana.

También se realizó una observación aplicada a los prestadores de servicio en el comedor universitario de la Universidad Estatal de Bolívar. En donde se pudo denotar que: 


\section{Gráfico1. Datos de la observación}

\begin{tabular}{|c|c|c|c|c|c|c|c|c|}
\hline Indicadores & \multicolumn{8}{|c|}{ Escalas } \\
\hline \multicolumn{3}{|l|}{ Intensidad de utilización de plástico } & Alta & 2 & Media & 2 & Baja & 1 \\
\hline $\begin{array}{l}\text { Tipo de productos que se sirven en } \\
\text { plásticos }\end{array}$ & $\begin{array}{l}\text { Preparados } \\
\text { en ese } \\
\text { momento }\end{array}$ & 4 & Procesados & 0 & $\begin{array}{l}\text { Productos } \\
\text { Frescos }\end{array}$ & 0 & $\begin{array}{l}\text { Bebidas y } \\
\text { jugos }\end{array}$ & 5 \\
\hline Tipo de contenedores que se utilizan & Plásticos & 5 & $\begin{array}{l}\text { De cartón/ } \\
\text { papel }\end{array}$ & 0 & $\begin{array}{l}\text { Loza y/o } \\
\text { cerámica }\end{array}$ & 5 & Otros & 0 \\
\hline \multicolumn{5}{|c|}{$\begin{array}{l}\text { Los contenedores poseen certificados de calidad que garanticen ser } \\
\text { biodegradables }\end{array}$} & Sí & 0 & No & 5 \\
\hline \multicolumn{5}{|l|}{ Son biodegradables } & Sí & 0 & No & 5 \\
\hline \multicolumn{3}{|l|}{ Lavan y reutilizan la vajilla y menaje } & Siempre & 1 & A veces & 4 & Nunca & 0 \\
\hline \multicolumn{3}{|c|}{ Clasifican la basura y desechos del comedor } & Sí & 0 & No & 5 & A veces & 0 \\
\hline $\begin{array}{l}\text { Cuánta basura plástica se produce } \\
\text { por día en el comedor universitario }\end{array}$ & $5 \mathrm{~kg}$ & 0 & $10 \mathrm{~kg}$ & 0 & $25 \mathrm{~kg}$ & 0 & $\begin{array}{l}\text { Más de } 25 \\
\mathrm{~kg}\end{array}$ & 5 \\
\hline
\end{tabular}

Fuente: Expendedores del comedor universitario

Elaboracón: Los investigadores

También se aplicaron encuestas, las cuales arrojaron los siguientes resultados.

Gráfico 2. Datos generales del ecuestado

EDAD

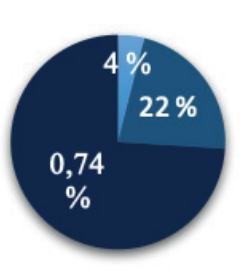

De 18 a 21 años

- De 22 a 25 años

- Más de 25 años
SEXO

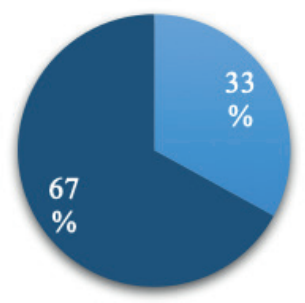

Eemenino Masculino
OCUPACIÓN ACTUAL

100

$\%$

- Estudiante $\square$ Empleado

Ninguno

Fuente: Usuarios del comedor universitario Elaboración: Los investigadores 
Al comedor universitario de la Universidad Estatal de Bolívar acuden estudiantes de 18 a 21 años, su afluencia mayoritaria es el género femenino.

La siguiente pregunta se centró en la satisfacción de los usuarios del comedor universitario, en donde:

Tabla 2. Satisfacción en el comedor universitario

\begin{tabular}{|l|l|l|}
\hline \multicolumn{1}{|c|}{ Variable } & \multicolumn{1}{|c|}{ Frecuencia } & \multicolumn{1}{c|}{$\begin{array}{c}\text { Porcentaje } \\
\text { (\%) }\end{array}$} \\
\hline A gusto & 345 & 94 \\
\hline Incomodo & 15 & 4 \\
\hline Indiferente & 7 & 2 \\
\hline Total & 367 & 100 \\
\hline
\end{tabular}

Fuente: Usuarios del comedor universitario

Elaboración: Los investigadores

El $94 \%$ de los encuestados se sienten a gusto con el servicio que reciben en el comedor universitario, a diferencia del $2 \%$ que expresa indiferencia en cuanto a la percepción del servicio.

Para la siguiente pregunta se buscó indagar sobre las motivaciones que tienen los usuarios para acudir al comedor universitario. En donde:

Tabla 3. Motivación

\begin{tabular}{|l|l|l|}
\hline \multicolumn{1}{|c|}{ Variable } & \multicolumn{1}{|c|}{ Frecuencia } & \multicolumn{1}{c|}{$\begin{array}{c}\text { Porcentaje } \\
\text { (\%) }\end{array}$} \\
\hline Precios de los productos & 195 & 53 \\
\hline Calidad de los productos & 60 & 16 \\
\hline Servicios que reciben & 75 & 20 \\
\hline Facilidad de llevarse los envases y alimentos & 37 & 10 \\
\hline Total & 367 & 100 \\
\hline
\end{tabular}

Fuente: Usuarios del comedor universitario

Elaboración: Los investigadores

El $53 \%$ de los usuarios están motivados a la compra por los precios de los productos, el $16 \%$ por la calidad, el $20 \%$ por los servicios que reciben y el $10 \%$ por la facilidad de portabilidad de los alimentos.

Basándonos en el segmento de usuarios que respondieron que les motiva la facilidad que los oferentes brindan para llevarse los productos de los bares, la siguiente pregunta se centró en conocer en qué tipo de recipientes prefiere recibir sus alimentos, para trasladarlos, a lo cual respondieron: 
Tabla 4. Preferencias

\begin{tabular}{|l|l|l|}
\hline \multicolumn{1}{|c|}{ Variable } & \multicolumn{1}{|c|}{ Frecuencia } & \multicolumn{1}{c|}{$\begin{array}{c}\text { Porcentaje } \\
\text { (\%) }\end{array}$} \\
\hline Cerámica / vidrio & 300 & 82 \\
\hline Plástico y sus derivados & 52 & 14 \\
\hline Cartón / Papel & 15 & 4 \\
\hline Total & 367 & 100 \\
\hline
\end{tabular}

Fuente: Usuarios del comedor universitario

Elaboración: Los investigadores

El $82 \%$ de los encuestados prefieren contenedores de cerámica o vidrio, mientras que el $14 \%$ manifiesta que prefiere envases de plástico y el $4 \%$ de cartón o papel.

Basándose en la pregunta anterior, se hace necesario conocer por qué los consumidores del comedor universitario prefieren el plástico como un elemento presente en la venta de los alimentos.

Tabla 5. Creencias del plástico

\begin{tabular}{|l|l|l|}
\hline \multicolumn{1}{|c|}{ Variable } & \multicolumn{1}{|c|}{ Frecuencia } & \multicolumn{1}{c|}{$\begin{array}{c}\text { Porcentaje } \\
\text { (\%) }\end{array}$} \\
\hline Saludable para el ser humano & 82 & 22 \\
\hline Perjudicial para su salud & 285 & 78 \\
\hline Total & 367 & 100 \\
\hline
\end{tabular}

Fuente: Usuarios del comedor universitario

Elaboración: Los investigadores

El $22 \%$ de encuestados consideran que el plástico es saludable para el ser humano, mientras que el $78 \%$ conocen lo perjudicial del uso indiscriminado del plástico para la salud de las personas.

También se busca conocer el pensamiento de los encuestados con relación a la naturaleza. Por lo que:

Tabla 6. Conocimiento del plástico

\begin{tabular}{|l|l|l|}
\hline \multicolumn{1}{|c|}{ Variable } & \multicolumn{1}{|c|}{ Frecuencia } & \multicolumn{1}{c|}{$\begin{array}{c}\text { Porcentaje } \\
\text { (\%) }\end{array}$} \\
\hline Amigables con el medio ambiente & 22 & 6 \\
\hline Contaminantes del medio ambiente & 345 & 94 \\
\hline Total & 367 & 100 \\
\hline
\end{tabular}

Fuente: Usuarios del comedor universitario Elaboración: Los investigadores

El $94 \%$ de los encuestados afirmaron que los plásticos son muy contaminantes del medio ambiente, manifestando el $6 \%$ que desconocían lo que provoca el plástico al medio ambiente y más bien expresaron ser amigables con el mismo. 

plástico.

La siguiente pregunta está basada en los productos que se sirven en algún tipo de

Tabla 7. Productos con envases plásticos

\begin{tabular}{|l|l|l|}
\hline \multicolumn{1}{|c|}{ Variable } & \multicolumn{1}{c|}{ Frecuencia } & \multicolumn{1}{c|}{ Porcentaje } \\
\hline Alimentos preparados en el momento & 112 & 31 \\
\hline Alimentos procesados & 120 & 33 \\
\hline Otros & 135 & 37 \\
\hline Total & 367 & 100 \\
\hline
\end{tabular}

Fuente: Usuarios del comedor universitario Elaboración: Los investigadores

Para el $31 \%$ de los encuestados los productos que más se venden son los preparados en ese momento, el 33 \% manifestó que son los procesados y el $37 \%$ señaló que son otros los productos con más venta.

La siguiente pregunta indaga a los consumidores si han observado alguna descripción de los contendores de alimento sobre sus propiedades técnicas, es decir, si son fácilmente degradables. A esta pregunta contestaron que:

Tabla 8. Descripción de los contenedores

\begin{tabular}{|c|c|c|}
\hline Variable & Frecuencia & $\begin{array}{c}\text { Porcentaje } \\
\text { (\%) }\end{array}$ \\
\hline Sí poseen & 105 & 29 \\
\hline No poseen & 262 & 71 \\
\hline Total & 367 & 100 \\
\hline
\end{tabular}

Fuente: Usuarios del comedor universitario

Elaboración: Los investigadores

El $29 \%$ de encuestados manifiestan que los contenedores de plástico sí tienen impreso en los envases sus propiedades biodegradables, mientas que el $71 \%$ lo niegan.

También se indagó si los consumidores han participado en algún programa o campaña que promueva la conservación del medio ambiente, las respuestas obtenidas fueron:

Tabla 9. Participación de campañas del medio ambiente

\begin{tabular}{|l|l|l|}
\hline \multicolumn{1}{|c|}{ Variable } & \multicolumn{1}{|c|}{ Frecuencia } & \multicolumn{1}{c|}{$\begin{array}{c}\text { Porcentaje } \\
\text { (\%) }\end{array}$} \\
\hline Sí & 127 & 35 \\
\hline No & 240 & 65 \\
\hline Total & 367 & 100 \\
\hline
\end{tabular}

Fuente: Usuarios del comedor universitario

Elaboración: Los investigadores 
El $65 \%$ de encuestados no ha participado en algún tipo de campaña de concientización del cuidado del medio ambiente. El $35 \%$ sí lo ha hecho.

Por lo que, para afirmar sobre el conocimiento se preguntó a los consumidores si conocen que en el comedor universitario existe contenedores para clasificar los desechos, en donde:

Tabla 10. Contenedores de clasificación de desechos

\begin{tabular}{|c|c|c|}
\hline Variable & Frecuencia & $\begin{array}{c}\text { Porcentaje } \\
\text { (\%) }\end{array}$ \\
\hline Sí & 10 & 3 \\
\hline No & 357 & 97 \\
\hline Total & 367 & 100 \\
\hline
\end{tabular}

Fuente: Usuarios del comedor universitario

Elaboración: Los investigadores

Para el $3 \%$ de los usuarios el comedor universitario sí cuenta con contenedores para clasificar los desechos, mientras que, el $97 \%$ manifiesta que no existe o desconocen la presencia de los mismos.

Para finalizar la encuesta a los consumidores, se les solicitó que escojan entre la mejor opción para conservar el medio ambiente. De lo cual:

Tabla 11. Conservación del medio ambiente

\begin{tabular}{|l|l|l|}
\hline \multicolumn{1}{|c|}{ Variable } & \multicolumn{1}{c|}{ Frecuencia } & \multicolumn{1}{|c|}{$\begin{array}{c}\text { Porcentaje } \\
\text { (\%) }\end{array}$} \\
\hline Recipientes a base de cartón y papel & 112 & 31 \\
\hline Utilizar loza y menaje de porcelana & 38 & 10 \\
\hline Disminuir el expendio de productos procesados & 10 & 3 \\
\hline Clasificar la basura y desechos del comedor & 97 & 26 \\
\hline Promover una cultura de conservación medio ambientalista & 110 & 30 \\
\hline Total & 367 & 100 \\
\hline
\end{tabular}

Fuente: Usuarios del comedor universitario

Elaboración: Los investigadores

El $31 \%$ de los encuestados está a favor de la propuesta de utilizar recipientes a base de cartón y papel; el $30 \%$ desearía que se promueva una cultura de conservación medio ambientalista; seguido del $26 \%$ que piensa que clasificar la basura y desechos del comedor es una buena alternativa; el $10 \%$ considera que sería bueno utilizar loza y menaje de porcelana; y, un $3 \%$ considera que se debe disminuir el expendio de productos procesados.

En el siguiente apartado también se exponen los resultados de la encuesta aplicada a los prestadores de servicio en el comedor universitario de la Universidad Estatal de Bolívar. Se preguntó sobre el grupo de clientes que resulta más conveniente atender, en donde: 
Tabla 12. Clientes convenientes

\begin{tabular}{|l|l|l|}
\hline \multicolumn{1}{|c|}{ Variable } & \multicolumn{1}{|c|}{ Frecuencia } & \multicolumn{1}{c|}{$\begin{array}{c}\text { Porcentaje } \\
\text { (\%) }\end{array}$} \\
\hline Estudiantes & 5 & 100 \\
\hline Empleados & 0 & 0 \\
\hline Particulares & 0 & 0 \\
\hline Total & 5 & 100 \\
\hline
\end{tabular}

Fuente: Expendedores del comedor universitario

Elaboración: Los investigadores

Por unanimidad los expendedores de alimentos concuerdan que es mejor realizar sus ventas a los estudiantes de la Universidad.

La siguiente pregunta se basó en conocer la motivación que tienen los expendedores, al momento de trabajar en el comedor universitario.

\section{Tabla 13. Motivación}

\begin{tabular}{|l|l|l|}
\hline \multicolumn{1}{|c|}{ Variable } & \multicolumn{1}{|c|}{ Frecuencia } & \multicolumn{1}{c|}{$\begin{array}{c}\text { Porcentaje } \\
\text { (\%) }\end{array}$} \\
\hline Mejorar su economía & 3 & 60 \\
\hline Aportar con la economía de otras personas & 1 & 20 \\
\hline Ofrecer un buen servicio & 0 & 0 \\
\hline Realizar prácticas de conservación ambiental & 1 & 10 \\
\hline Total & 5 & 100 \\
\hline
\end{tabular}

Fuente: Expendedores del comedor universitario

Elaboración: Los investigadores

El $60 \%$ respondió que la motivación que tiene para trabajar en el comedor de la Universidad Estatal de Bolívar es por mejorar su economía, les siguen el grupo de personas cuya motivación es aportar a la economía de su hogar, el $1 \%$ restante manifiesta que lo haace por realizar prácticas de conservación ambiental.

Para conocer más sobre la situacion de la forma de expender los productos del comedor, se pregunto en qué tipo de recipientes prefiere servir los alimentos. En donde:

Tabla 14. Preferencias

\begin{tabular}{|c|c|c|}
\hline Variable & Frecuencia & $\begin{array}{c}\text { Porcentaje } \\
\text { (\%) }\end{array}$ \\
\hline Cerámica / vidrio & 3 & 60 \\
\hline Plástico y sus derivados & 2 & 40 \\
\hline Cartón / papel & 0 & 0 \\
\hline Total & 5 & 100 \\
\hline
\end{tabular}

Fuente: Expendedores del comedor universitario

Elaboración: Los investigadores 
Los expendedores muestran una preferencia por servir los productos de sus locales comerciales, en recipientes de cerámica o vidrio $(60 \%)$ y el $40 \%$ preferiría seguir expendiendo en recipientes de plásticos y sus diversos derivados.

También se indagó sobre la creencia sobre los alimentos servidos en contenedores de plástico, de lo cual los expendedores respondieron que:

\section{Tabla 15. Creencias}

\begin{tabular}{|l|l|l|}
\hline \multicolumn{1}{|c|}{ Variable } & \multicolumn{1}{c|}{ Frecuencia } & \multicolumn{1}{c|}{$\begin{array}{c}\text { Porcentaje } \\
\text { (\%) }\end{array}$} \\
\hline Saludable para el ser humano & 0 & 0 \\
\hline Perjudicial para su salud & 5 & 100 \\
\hline Total & 5 & 100 \\
\hline
\end{tabular}

Fuente: Expendedores del comedor universitario Elaboración: Los investigadores

Un $100 \%$ respondió que servir la comida en contenedores de plástico es perjudicial para la salud de los consumidores.

Se realizó la pregunta, si los expendedores de alimentos consideran que el plástico tiene relación con el cuidado ambiental, en donde:

Tabla 16. Conocimiento del plástico

\begin{tabular}{|l|l|l|}
\hline \multicolumn{1}{|c|}{ Variable } & \multicolumn{1}{c|}{ Frecuencia } & \multicolumn{1}{c|}{ Porcentaje } \\
\hline Amigable con el medio ambiente & 0 & 0 \\
\hline Contaminante para el medio ambiente & 5 & 100 \\
\hline Total & 5 & 100 \\
\hline
\end{tabular}

Fuente: Expendedores del comedor universitario Elaboración: Los investigadores

El $100 \%$ de los encuestados consideran que la utilización del plástico también es un contaminante para el medio ambiente (naturaleza).

La siguiente pregunta se basó en conocer el tipo de productos que se sirven en contenedores de plástico.

Tabla 17. Productos con plástico

\begin{tabular}{|l|l|l|}
\hline \multicolumn{1}{|c|}{ Variable } & \multicolumn{1}{|c|}{ Frecuencia } & \multicolumn{1}{c|}{$\begin{array}{c}\text { Porcentaje } \\
\text { (\%) }\end{array}$} \\
\hline Alimentos procesados en el momento & 4 & 80 \\
\hline Alimentos procesados & 0 & 0 \\
\hline Todo tipo de productos & 1 & 20 \\
\hline Total & 5 & 100 \\
\hline
\end{tabular}

Fuente: Expendedores del comedor universitario

Elaboración: Los investigadores 
El $80 \%$ de los expendedores sirve los alimentos procesados en el momento, mientras que, el $1 \%$ utiliza el plástico para todo tipo de productos.

También se indagó sobre el conocimiento de las características físicas / químicas de los contenedores de alimentos.

Tabla 18. Productos con plástico

\begin{tabular}{|c|c|c|}
\hline Variable & Frecuencia & $\begin{array}{c}\text { Porcentaje } \\
\text { (\%) }\end{array}$ \\
\hline Son biodegradables & 1 & 20 \\
\hline No son biodegradables & 0 & 0 \\
\hline Desconoce & 4 & 80 \\
\hline Total & 5 & 100 \\
\hline
\end{tabular}

Fuente: Expendedores del comedor universitario

Elaboración: Los investigadores

Para el $20 \%$ de los encuestados los productos de plástico que utiliza son biodegradables, mientras que el $80 \%$ manifiesta desconocer el tipo de contenedor de plástico que maneja.

Para complementar el estudio, también se indagó si los expendedores de productos en el comedor universitario han participado en algún programa o campaña que promueva la conservación del medio ambiente. En donde:

Tabla 19. Participación de campañas del medio ambiente

\begin{tabular}{|l|l|l|}
\hline \multicolumn{1}{|c|}{ Variable } & \multicolumn{1}{|c|}{ Frecuencia } & \multicolumn{1}{c|}{$\begin{array}{c}\text { Porcentaje } \\
\text { (\%) }\end{array}$} \\
\hline Sí & 0 & 0 \\
\hline No & 5 & 100 \\
\hline Total & 5 & 100 \\
\hline
\end{tabular}

Fuente: Expendedores del comedor universitario

Elaboración: Los investigadores

El $100 \%$ de encuestados no ha participado en alguna campaña de conservación del medio ambiente. Por lo que, se hizo necesario preguntar si cada expendedor realiza alguna actividad para la clasificación de desechos de basura. De lo cual:

Tabla 20. Clasificación de desechos

\begin{tabular}{|l|l|l|}
\hline \multicolumn{1}{|c|}{ Variable } & \multicolumn{1}{c|}{ Frecuencia } & \multicolumn{1}{c|}{ Porcentaje } \\
\hline $\mathrm{Si}$ & 1 & 20 \\
\hline $\mathrm{No}$ & 4 & 80 \\
\hline Total & 5 & 100 \\
\hline
\end{tabular}

Fuente: Expendedores del comedor universitario

Elaboración: Los investigadores 
El $20 \%$ si clasifica la basura, mientras que el $80 \%$ de encuestados no realiza ninguna actividad de clasificación de los residuos.

Finalmente, se preguntó sobre la propuesta que mas factibilidad consideran los expendedores, para poder conservar el medio ambiente. En donde:

Tabla $N^{\circ}$ 21. Conservación del medio ambiente

\begin{tabular}{|l|l|l|}
\hline \multicolumn{1}{|c|}{ Variable } & \multicolumn{1}{|c|}{ Frecuencia } & \multicolumn{1}{|c|}{$\begin{array}{c}\text { Porcentaje } \\
(\%)\end{array}$} \\
\hline Recipientes a base de cartón y papel & 0 & 0 \\
\hline Utilizar loza y menaje de porcelana & 1 & 20 \\
\hline Disminuir el expendio de productos procesados & 0 & 0 \\
\hline Clasificar la basura y desechos del comedor & 3 & 60 \\
\hline Promover una cultura de conservación medio ambientalista & 1 & 20 \\
\hline Total & 367 & 100 \\
\hline
\end{tabular}

Fuente: Expendedores del comedor universitario

E : Los investigadores

Como indica la tabla, el $60 \%$ considera que una buena medida es clasificar la basura y los desechos del comedor, el $20 \%$ utilizar loza y menaje de porcelana y promover una cultura de conservación medio ambientalista.

\section{CONCLUSIONES}

\section{Discusión}

El tema de contaminación ambiental es de preocupación latente y consituye un tema de interés desde los productores o generadores de basura, consumidores, recicladores, entre otros. Su importancia radica en la necesidad de entregar una propuesta de solución viable, sostenible y acorde con las exigencias de los consumidores, la necesidad de los prestadores de servicio y la costumbre de una comunidad joven (estudiantil) que desea vivir con la modernidad, conservando el equilibrio medio ambiental.

En el comedor universitario de la Universidad Estatal de Bolívar, la mayor parte que acuden son estudiantes de 18 a 21 años, su afluencia mayoritaria es de género femenino; quienes sienten satisfacción cuando acuden a dicho lugar. Entre las causas que les motivan a ir al comedor universitario, es por el precio de los productos, por el servicio que reciben, por la calidad de los productos y porque se puede llevar los envases y alimentos.

Al indagar sobre las preferencias de los recipientes para el consumo, señalaron que se sienten más cómodos utilizando cerámica y vidrio, sin descartar el consumo en recipientes de cartón o papel; así demostrando el nivel de desinformación que los usuarios poseen sobre la influencia del plástico en la contaminación ambiental, necesitándose una estrategia adecuada de sensibilización sobre este perjuicio. A pesar de que, los encuestados manifestaron que los alimentos servidos en plásticos son perjudiciales para su salud, porque contienen materiales químicos que afectan a los organismos vivos, ríos, mares y sobre todo la vida en el plantea. 
Se manifiesta que los plásticos son contaminantes del medio ambiente, siendo un factor constante entre los usuarios, lo que determina la ausencia de concientización sobre la contaminación ambiental y en especial los daños que provoca el plástico al llegar a la naturaleza sin ningún tipo de control o tratamiento.

Al indagar sobre la identificación o etiquetas de los productos en función de su degradación, la mayor cantidad de usuarios mencionaron que los contenedores de alimentos no poseen ningún tipo de descripción sobre las propiedades físicas y químicas de los envases, mismos que manifiestan que no han participado en ningún programa o campaña que promueva la conservación del medio ambiente, por lo que es urgente y necesario propiciar espacios de concientización a los usuarios del comedor universitario sobre distintas formas de evitar la contaminación ambiental, exigiendo o inclinándose a no consumir en gran cantidad productos en envases plásticos.

Otro punto es la ausencia de contenedores para clasificar los desechos en el comedor universitario, lo que implica un inadecuado tratamiento de la basura que se concentra en un solo lugar y que definitivamente inciden a la contaminación. Tomando en cuenta que, en relación con las opciones como propuesta para conservar el medio ambiente, la mayoría se inclinan por utilizar recipientes a base de cartón/papel y promover una cultura de conservación medioambientalista, y con menor incidencia están el clasificar la basura y desechos del comedor, utilizar loza y menaje de porcelana, disminuir el expendio de productos procesados.

Para los proveedores del servicio de alimentación es más conveniente atender solo a los estudiantes, a quienes prefieren servir los alimentos en cerámica o vidrio, y también en recipientes de plástico; considerándose estas prácticas de servicio como el principal factor de contaminación para el medio ambiente, por lo que, definitivamente se les debe capacitar sobre formas alternas de uso en el lugar. A pesar de que la creencia sobre las implicaciones del plástico en el servicio de alimentación, la mayoría dicen que son perjudiciales para la salud; sin embrago, persiste la costumbre, contribuyendo de manera deliberante y poco responsable a la contaminación del ambiente.

Al preguntar a los encuestados si han participado en algún programa o campaña que promueva la conservación del medio ambiente, los expendedores dijeron que no. Este indicador demuestra la necesidad de instruir y capacitar a los proveedores del servicio de alimentación del comedor universitario sobre una nueva propuesta que regule el uso de contenedores de alimentos a base de materiales biodegradables y amigables con el medio ambiente, la clasificación y reciclaje de desechos y otras que permitan se sustituyan el plástico y sus derivados para evitar efectos contaminantes que son irreversibles una vez que llegan a su destino común.

Sobre las características fisicoquímicas de los contenedores de alimentos se obtuvo que la mayoría de ellos dijeron desconocerlas. Y apenas uno de cinco dijeron que son biodegradables. En relación con la práctica de clasificación de los desechos y basura, se obtuvo que la mayoría dijeron que no se la realiza en el comedor universitario ni fuera de él.

Para determinar cuáles serían las propuestas para conservar el medio ambiente, la mayoría se orientó hacia la clasificación de la basura y desechos del comedor, la utilización de loza y menaje de porcelana, y la promoción de una cultura de conservación medioambientalista. Con estos resultados se justifica y hace evidente la necesidad de promover estrategias de venta y comercialización de sus productos ligados a una cultura medio ambientalista que evite la contaminación del medio y se culturice a los usuarios y proveedores del servicio de alimentación para evitar el consumo de plásticos por otros que sean biodegradables y amigables. 
Al realizar la observación de los procesos diarios, los materiales que utilizan y prácticas para eliminación de basura y desechos del comedor universitario, se obtuvieron los siguientes resultados:

- En relación con la intensidad de uso de plásticos como contenedores y para servir los alimentos se estima que es alto su consumo, ya que se sirven toda clase de comidas preparadas en ese momento, aquellas que vienen procesadas a nivel industrial y otros que aparentemente economizan el tiempo de los empleados.

- Al hablar de los contenedores de alimentos, platos, vasos y utensilios no cuentan con una certificación de fabricación que describan sus características físicas o químicas para garantizar que sean biodegradables o no.

- En pocos lugares dentro del comedor universitario se observó que sirven los alimentos en platos de cerámica y vidrio, que luego son lavados y reutilizados. Mientras que la mayoría de ellos se dedicaron a servir toda clase de productos en fundas, cucharas, palillos y vasos plásticos, sin contar con los envases que vienen los productos procesados como papas, gaseosas, aguas, entre otros.

- Todos aquellos desperdicios llegan a un contenedor en común, en donde no se clasifican plásticos, cartón-papel y vidrio, cuyo destino es el basurero común de la institución.

- Diariamente se genera un contenedor de basura cuyas medidas son: largo 1265 $\mathrm{mm} \times$ ancho $780 \mathrm{~mm}$ x alto: $1270 \mathrm{~mm}$. Con una capacidad de carga aproximada de $120 \mathrm{~kg}$ de basura en el comedor universitario, lo que da como resultado de $600 \mathrm{~kg}$ a la semana, y $2.4 \mathrm{Tm}$. al mes de basura plástica solo en el comedor. Lo que podría suponerse $24 \mathrm{Tm}$ al año que contaminan el medio ambiente de forma irremediable.

Por otro lado, la mayoría de los proveedores del servicio de alimentos no son conscientes sobre la necesidad de cambiar las estrategias de venta de sus productos cuidando de mejor manera el medio ambiente, para así evitar y reducir las prácticas contaminantes. En donde el cartón/papel no son opciones de uso. Esta poca afinidad por el uso de este material es limitada porque se piensa que el plástico es más económico que usar papel, porque en el medio muy poco se encuentran recipientes de cartón-papel biodegradables y estos elevan sus costos de producción y disminuyen sus utilidades, pero nada más erróneo si se piensa en los beneficios que se obtendrán a largo plazo, con un ambiente más sano y libre de contaminantes.

Estos resultados alarmantes pasan desapercibidos por los usuarios y proveedores del servicio de alimentación en el lugar, al igual que las autoridades de la institución patrocinadora que no ha fijado su atención en las prácticas de consumo de plásticos y sus derivados como un factor contaminante del medio ambiente.

Ante esta realidad, surge la necesidad de crear conciencia y es preciso hacerlo desde el lugar de convivencia en el que nos desarrollamos, con la esperanza de concientizar al mayor número de personas a suprimir el uso de plásticos y sus derivados por otros que sean biodegradables y amigables con el medio ambiente, sustituyendo estrategias de mercado y comercialización de productos con una cultura medio ambientalista.

\section{PROPUESTA}

1. Campaña de Marketing Social.

2. Diseño de envases a base de cartón que sean biodegradables. 
3. Elaboración y entrega de un tríptico informativo sobre la influencia del plástico en la contaminación ambiental.

4. Socialización de información a los prestadores de servicios de alimentos y usuarios.

5. Difusión de resultados.

\section{RESULTADOS ESPERADOS}

- Incrementar el uso de recipientes biodegradables.

- Adaptar al consumidor y prestadores de servicios al manejo y utilización de recipientes biodegradables.

- Disminuir el índice de contaminación ambiental por plásticos.

- Incentivar a los prestadores de servicios y usuarios a abstenerse de utilizar plásticos y optar por envases de cartón biodegradable amigables con el medio ambiente.

\section{CONCLUSIONES}

Se identificaron como las principales formas de contaminación al medio ambiente el uso indiscriminado de plásticos en los productos de consumo, preparados en ese momento y todos los que se expenden de forma industrial, no existe un solo lugar que se inclinen por el uso de envases, platos, cucharas y otros que sean biodegradables o reutilizables.

Es preciso ejecutar programas de concientización a los usuarios y proveedores del servicio de alimentación sobre la importancia de preservar el medio ambiente, a través de campañas de concientización y la formación de conductas medioambientalistas que protejan los recursos naturales, procurando la utilización de productos amigables con el medio ambiente.

Se ha diseñado una propuesta alternativa de marketing que busca sustituir el uso de plásticos por envases de cartón y papel, además, que se promueva la clasificación de los desechos para el reciclaje de los mismos, promocionar y adquirir productos que garanticen su biodegradación y, sobre todo, que sean usuarios y proveedores responsables de sus acciones frente a la naturaleza.

\section{BIBLIOGRAFÍA}

Aguilar, L. (2016). 1 de octubre. Recuperado de http://contaminacion-ambiente.blogspot.com/2006/10/ que-es-la-contaminacion-ambiental.html

American Marketing Association. (2016). 1 de enero. Recuperado de https://www.ama.org/

BBC. (2018). Los 10 países que más y menos basura generan en América Latina (y cómo se sitúan a nivel mundial), 9 de octubre. Recuperado de https://www.bbc.com/mundo/noticias-45755145

Chicaiza, J., y González, E. (2016). Universidad Estatal de Guayaquil. 1 de enero. Recuperado de Análisis de los desechos de botellas: http://repositorio.ug.edu.ec/bitstream/redug/17450/1/TESIS\%20 JOSELYN\%20CHIC AIZA\%20Y\%20EVELYN\%20GONZALEZ\%20FINAL\%20PDF.pdf

Díaz, C. (2018). Investigación cualitativa y análisis de contenido temático. Orientación intelectual de revista. Universum. Revista General de Información y Documentación., 28 (1): 119-142.

Estévez, R. (2019). Ambientum, 28 de agosto. Recuperado de https://www.ambientum.com/ambientum/residuos/contaminacion-plastico.asp

Eva, M. (2009). Cosmoteca, 1 de enero. Recuperado de https://www.consumoteca.com/bienestar-ysalud/medio-ambiente/reutilizacion/ 
Góngora, L. (2014). Mercadotecnia social: una herramienta necesaria para la promoción de salud. MEDISAN, 18 (5): 684-694.

Kottler, P. (2006). Fundamentos de Marketing. Madrid, España: Pearson Educaction.

Linea Verde Ceuta. (2018), 1 de enero. Recuperado de http://www.lineaverdeceutatrace.com/lv/consejos-ambientales/contaminantes/Que-es-la-contaminacion-ambiental.asp

Morán, S. (2018). Plan V hacemos noticias, 23 de julio. Recuperado de https://www.planv.com.ec/ historias/sociedad/basura-numeros-rojos-ecuador

Paredes, G. (2009). Cómo desarrollar una tesis. Guaranda, Ecuador. S\&S.

Pérez, J., y Gardey, A. (2013). Definición, 1 de enero. Recuperado de https://definicion.de/reciclaje/

Pérez, J., y Merino, M. (2008). Concepto, 1 de enero. Recuperado de https://definicion.de/contaminacion/

QuestionPro. (2019). Diseño de investigación y tipos que existen, 1 de enero Recuperado de https:// www.questionpro.com/blog/es/diseno-de-investigacion/

Rodríguez, D. (2018). Recuperado de Investigación aplicada, 1 de enero. https://www.lifeder.com/ investigacion-aplicada/

Salinas, J. (2011). Definición y Clasificación de Plásticos. Recuperado de Tecnología de manera divertida, 1 de febrero. http://tecnologmota.blogspot.com/2011/02/definicion-y-clasificacionde-plasticos.html

Vargas, Z. (2009). La investigación aplicada: una forma de conocer las realidades con evidencia científica. Revista Educación 33 (1): 155-165.

Vera, J., y Villegas, V. (1997). El fenómeno de la comunicación social. Revista Archivo Médico de Camagüey, 1 (1):13. 